\title{
CORRELATIVE FIRING PATTERNS OF SEROTONERGIC NEURONS IN RAT DORSAL RAPHE NUCLEUS ${ }^{1}$
}

\author{
REX Y. WANG ${ }^{*, 2}$ AND GEORGE K. AGHAJANIAN $\ddagger$ \\ ${ }^{*}$ Department of Pharmacology, St. Louis University, St. Louis, Missouri 63104 and $\ddagger$ Departments of Psychiatry and \\ Pharmacology, Yale University, New Haven, Connecticut 06508
}

Received May 15, 1981; Revised September 18, 1981; Accepted September 21, 1981

\begin{abstract}
In this study, local neuronal interactions in the midbrain dorsal raphe nucleus (DRN) were analyzed by the use of autocorrelation and cross-correlation histograms. The autocorrelograms of serotonin (5-hydroxytryptamine; 5-HT)-containing cells showed an initial trough and subsequent periodicity which corresponded to their regular rhythmic firing pattern. Cross-correlograms revealed that all adjacent 5-HT neurons recorded from single micropipettes displayed complicated patterns of functional interactions. The interactions could be grouped into the following categories: (1) synchronization (interspike intervals $<10 \mathrm{msec}$ ), (2) synchronization and direct inhibition, and (3) synchronization and mutual inhibition. In contrast, cross-correlograms generated from adjacent neuronal pairs consisting of one 5-HT cell and one non-5-HT neuron or from non-adjacent 5-HT neuronal pairs usually failed to show functional interactions. The results of the present study are consistent with previous findings of a powerful auto- and mutual regulatory system for 5-HT neurons in the DRN.
\end{abstract}

The dorsal raphe nucleus (DRN) possesses the largest clusters of serotonin (5-hydroxytryptamine; 5 -HT)-containing neurons in the brain (Dahlström and Fuxe, 1965). Electrophysiological studies have shown that 5-HT neurons in the DRN are strongly inhibited following antidromic activation of the ascending 5-HT pathway (Wang and Aghajanian, 1977b, 1978). Theoretically, these inhibitory effects could be mediated via recurrent 5-HT axon collaterals and/or dendrodendritic junctions. This view is supported by the facts that: (1) the inhibition induced by antidromic stimulation of the 5-HT pathway depends upon the integrity of 5-HT fibers as it is prevented when 5-HT axons in the ventromedial tegmentum are destroyed by 5,7-dihydroxytryptamine (Wang and Aghajanian, $1977 \mathrm{~b})$, (2) 5-HT neurons are readily inhibited by microiontophoretically applied 5-HT (Aghajanian et al., 1972; Haigler and Aghajanian, 1974; Wang and Aghajanian, 1977b), (3) the DRN contains one of the heaviest concentrations of 5-HT receptors in the brain (Bennett and Aghajanian, 1974; Bennett and Snyder, 1975, 1976;

\footnotetext{
'We thank Nancy Margiotta and Annette Zimnewicz for their excellent technical assistance and B. Barnes for typing the manuscript. This work was supported by United States Public Health Service Grants MH-34424 and MH-00378 to R. Y. W. and Grants MH-17871 and MH-14459 to G. K. A. and by the State of Connecticut.

${ }^{2}$ To whom correspondence should be addressed at Department of Pharmacology, St. Louis University School of Medicine, 1402 South Grand Blvd., St. Louis, MO 63104.
}

Lovell and Freedman, 1976; Fillion et al., 1978; Peroutka and Snyder, 1979; Young and Kuhar, 1980), and (4) high affinity 5-HT uptake (Shaskan and Snyder, 1970; Donzanti and Warwick, 1980) and release of $5-\left[{ }^{3} \mathrm{H}\right] \mathrm{HT}$ (Kerwin and Pycock, 1979) have been demonstrated in the brain slices from the rat raphe area. Moreover, interactions between dendrite bundles in the DRN have been suggested on the basis of Golgi and histofluorescence studies (Felten et al., 1980). However, electron microscopic autoradiographic studies have failed to demonstrate the labeling of axosomatic or axodendritic junctions by $5-\left[{ }^{3} \mathrm{H}\right] \mathrm{HT}$ within the neuropil of the DRN (Baraban and Aghajanian, 1981; Descarries et al., 1979).

The aim of the present study was to elucidate further the nature of functional interactions among 5 - HT cells in the DRN by the use of autocorrelation and crosscorrelation techniques; the latter techniques can be useful in disclosing the mechanisms by which the firing of one neuron influences the probability of the firing of the other neuron (Moore et al., 1966, 1970; Perkel et al., $1967 \mathrm{a}, \mathrm{b})$. Although many studies have been devoted to the investigation of pharmacological or electrophysiological manipulations of single 5-HT neurons in the DRN (e.g., see Aghajanian and Wang, 1978 for review), the information concerning the local interactions among multiple neurons within the nucleus is still lacking. The latter knowledge is important because the DRN is not a homogeneous entity as it consists of both 5-HT and non-5HT neurons (Aghajanian et al., 1978; Descarries et al., 
1979; Belin et al., 1979; McGeer et al., 1979; Gamrani et al., 1979; Steinbusch et al., 1980).

\section{Materials and Methods}

Experimental subjects and single unit recordings. Male Sprague-Dawley albino rats (Charles River; 200 to $300 \mathrm{gm})$ were anesthetized with chloral hydrate $(400 \mathrm{mg} /$ $\mathrm{kg}$, i.p.); additional injections were given as needed. The animals were mounted in a stereotaxic apparatus and a burr hole was drilled over the DRN with the center at $0.5 \mathrm{~mm}$ anterior to the lambda, in the midline. In addition, two low cerveau isole rats were prepared under the halothane anesthesia. The detailed procedures for brain stem transection have been described (Wang and Aghajanian, 1977a). During the experiments, wound edges and pressure points were infiltrated by a solution of $2 \%$ mepivacaine hydrochloride.

Single barrel micropipettes were filled with $2 \mathrm{M} \mathrm{NaCl}$ saturated with Fast Green (Fischer). The electrodes typically had an impedance of 3 to 5 megohms when measured at $60 \mathrm{~Hz}$ in vitro. In another series of experiments, extracellular action potentials were recorded by special electrodes which were constructed by cementing a single barrel recording micropipette in parallel with another single barrel micropipette but with $100-\mu \mathrm{m}$ tip separation (Wang and Aghajanian, 1977b). Electrodes were lowered through the burr hole into the midbrain raphe region with a hydraulic microdrive system. Electrode signals were passed through a high input impedance amplifier, monitored on an oscilloscope, and stored on magnetic tape for subsequent analysis. When the recording electrode was lowered into the midbrain central gray, passing through the midline, a zone of electrical silence, often with $60 \mathrm{~Hz}$ noise (which corresponded to the depth of the cerebral aqueduct), was encountered just dorsal to the DRN. Attempts were made to isolate more than one cell on each electrode. At the end of each experiment, recording sites were marked by passing $20 \mu \mathrm{A}$ of cathodal current through the recording barrel for $15 \mathrm{~min}$, depositing a green spot at the electrode tip (Thomas and Wilson, 1965). Rats then were perfused with $10 \%$ formalin. The precise location of the electrode tip was confirmed by histological examination and compared with the previously established location of 5-HT-containing neurons in the DRN.

Data analysis. The magnetic tape was replayed through a window discriminator and monitored on an oscilloscope; the standard outputs of Schmitt triggers of the window discriminator were led into a Nicolet computer (Med 80) to generate autocorrelation and crosscorrelation histograms, which then were plotted on paper via a X-Y recorder. The data were retained for statistical analysis only when individual cells maintained a signalto-noise ratio of $3: 1$ or better. In the case of two cells recorded simultaneously from the same micropipette, the signal-to-noise criterion was relaxed occasionally to $2: 1$ for the smaller cell.

\section{Results}

General description. A total of 94 cells were recorded in the DRN. Cross-correlation analyses were obtained from 33 pairs of simultaneously recorded spike trains, 24 pairs of adjacent cells were recorded with single micro- pipettes, and 9 pairs of non-adjacent cells were obtained from specialized electrodes. The latter consisted of two micropipettes with a tip separation of approximately 100 $\mu \mathrm{m}$.

5-HT cells in the DRN were identified tentatively by their waveform (i.e., a predominant positive, then a negative wave) and by their slow, regular spontaneous discharge rates $(0.5$ to $3 \mathrm{~Hz})$. This firing pattern has been demonstrated by combined single cell recording and fluorescence histochemical methods to be characteristic for 5-HT-containing neurons but not for non-5-HT-containing cells in the DRN (Aghajanian and Haigler, 1974; Aghajanian et al., 1978). The identification of the slow, regular firing units in the DRN as 5-HT neurons has been confirmed by use of antidromic stimulation of the ascending 5-HT pathway, collision tests, and blockade of 5-HT transmission by 5,7-dihydroxytryptamine (Wang and Aghajanian, 1977c).

Autocorrelation. A typical slow, regular spike train is shown in Figure $1 A$; Figure $1 B$ is the autocorrelation histogram (autocorrelogram) of the same cell showing an initial prolonged quiescent period (trough) followed by periodic fluctuations, with equally spaced peaks appearing approximately every $460 \mathrm{msec}$. Since an autocorrelogram specifies the probability of encountering any spikes as a function of time after a given spike, the histogram implies that, for any spike in the discharge sequences, there is a high probability of finding the successive spikes at intervals of $460 \mathrm{msec}$. This silhouette of a 5-HT neuron's autocorrelogram is characteristic of cells with a regular or rhythmic firing pattern (Moore et

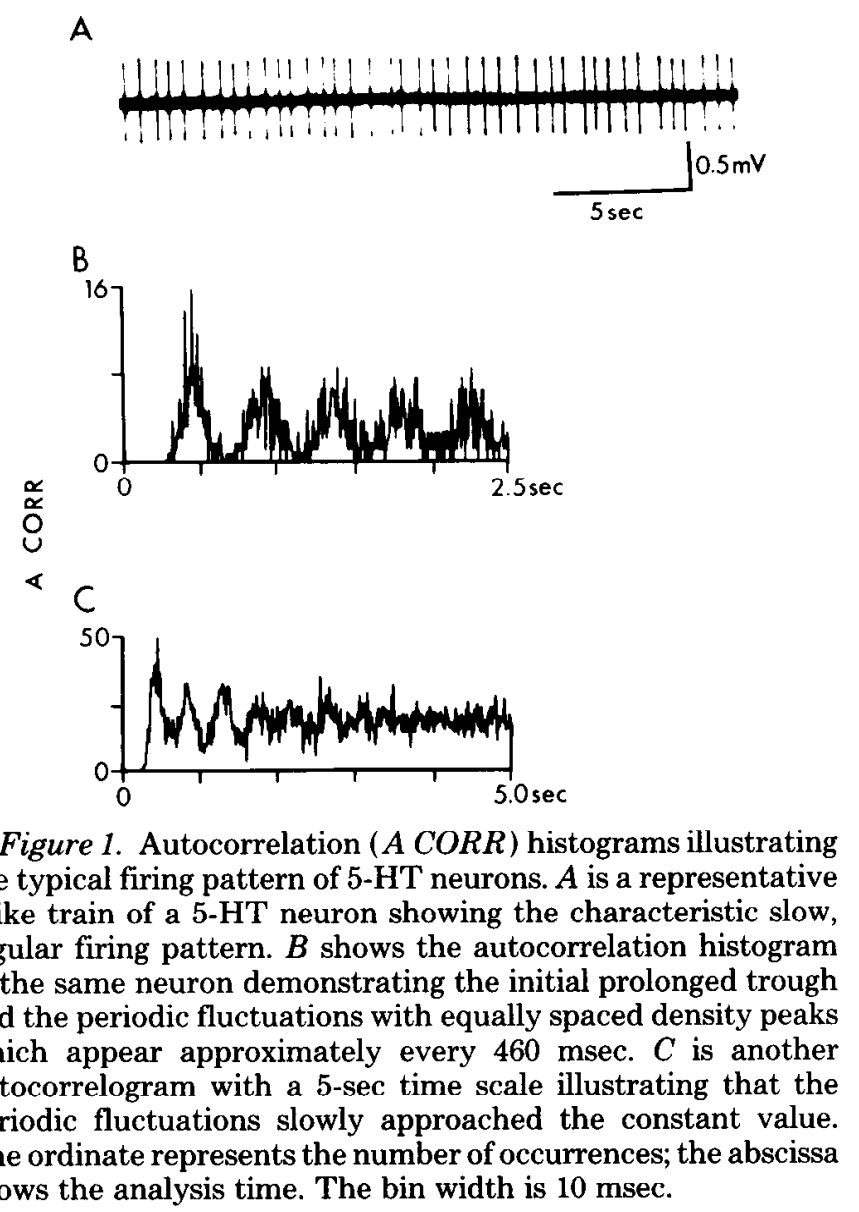


al., 1970). A property of the autocorrelation is that it "flattens out" to a constant value; in general, this limit is approached slowly for pacemaker neurons and more rapidly for spike trains with greater variation in interval length. Figure $1 C$ is an autocorrelogram of a 5-HT neuron with a 5-sec time scale showing that the periodic density peaks slowly approach the constant value. All autocorrelation histograms generated from presumed 5-HT cells exhibited this pattern; the cells with higher discharge rates $(2$ to $3 \mathrm{~Hz})$ tended to discharge more regularly with less variations of interspike intervals, and thus, their autocorrelograms usually took a longer time to level off. It should be noted that the characteristic slow, regular firing pattern of 5-HT cells was not the result of chloral hydrate anesthesia as the autocorrelograms generated from 5 -HT cells $(n=3)$ in unanesthetized low cerveau isole rats were very similar to the pattern described above.

The autocorrelograms of non-5-HT neurons in the DRN showed completely different patterns (e.g. Fig. 4A) from those of 5-HT neurons; they did not have the initial prolonged trough nor did they have periodic fluctuations with equally spaced peaks. The result was expected since non-5-HT cells in the DRN usually have much higher discharge rates and/or irregular activities.

Cross-correlation. To evaluate the probability of the correlated firing of two neurons, cross-correlation histograms were constructed. Figure $2 A$ shows a typical example of spike trains of an adjacent 5-HT neuronal pair recorded from a single microelectrode; the autocorrelogram of each neuron and the cross-correlation histogram generated from spike trains of this neuronal pair are shown in Figure 2, $B, C$, and $D$, respectively. The crosscorrelogram provides a measure of the probability of encountering a spike in train B (small cell) as a function of time before (left half of the histogram) or after (right half) a spike in train A (large cell); the probability of encountering a B spike by chance is related to the background activity which is proportional to the mean firing rates of $A$ and $B$ neurons. A remarkable correlation peak

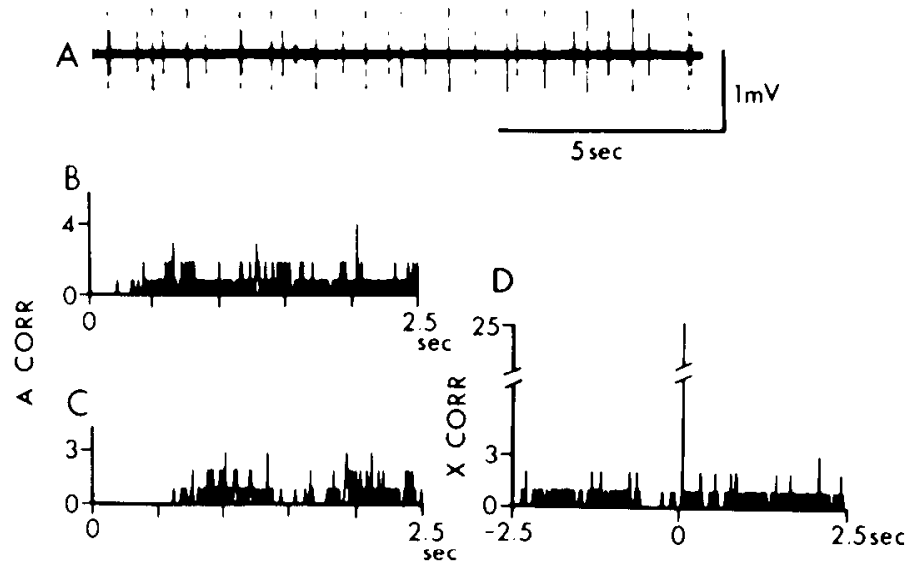

Figure 2. A typical example of inhibitory interactions among adjacent 5-HT neurons in the DRN. A segment of spike trains consisting of a pair of 5-HT neurons is shown in $A, B$ and $C$ are autocorrelation ( $A C O R R$ ) histograms generated from the large (cell $\mathrm{A}$ ) and the small (cell $\mathrm{B}$ ) cells, respectively. $D$ is a crosscorrelation ( $X$ CORR) histogram generated from the two spike trains showing synchrony and "direct inhibition." close to the origin was found in this adjacent 5-HT neuron pair, indicating that their firing was often in synchrony (the intervals between spikes were less than 10 msec; Fig. $2 A$ ). In addition to the synchronization, there was possibly a "direct inhibition" exerted from cell B onto cell A. All cross-correlograms obtained from spike trains of adjacent 5-HT neuronal pairs suggested complicated inhibitory interactions among adjacent 5-HT neurons. In general, the different interaction patterns could be grouped into three major categories which are summarized in Table $I$ and illustrated in Figure 3. In

TABLE I

Patterns of interaction among adjacent 5-HT neuron pairs and other neuron pairs in the DRN

\begin{tabular}{|c|c|c|c|}
\hline \multirow[t]{2}{*}{ Pattern of Interaction } & \multicolumn{2}{|c|}{ Adjacent Neuron Pairs } & \multirow{2}{*}{$\begin{array}{c}\text { Non-adja- } \\
\text { cent Neuron } \\
\text { Pairs } \\
5-H T-5-H T\end{array}$} \\
\hline & 5-HT-5-HT & 5-HT-non-5-HT & \\
\hline \multicolumn{4}{|l|}{ Correlated interaction } \\
\hline Synchrony & 5 & 0 & 0 \\
\hline $\begin{array}{l}\text { Synchrony and direct } \\
\text { inhibition }\end{array}$ & 10 & 0 & 1 \\
\hline $\begin{array}{l}\text { Synchrony and mutual } \\
\text { inhibition }\end{array}$ & 6 & $\underline{0}$ & 0 \\
\hline Total & 21 & $0^{a}$ & $1^{a}$ \\
\hline No correlation & 0 & 3 & 8 \\
\hline
\end{tabular}

"Compared to adjacent 5-HT-5-HT neuron pairs, the difference between the proportion of neuronal pairs showing correlated interaction is highly significant ( $p<0.005$ for both cases; Fisher's test for significance for $2 \times 2$ tables).
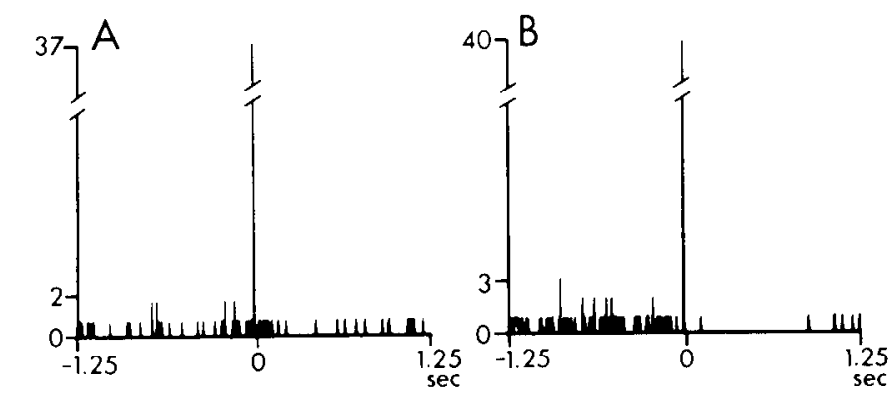

\begin{tabular}{l}
$\alpha$ \\
\hdashline \\
\hdashline
\end{tabular}

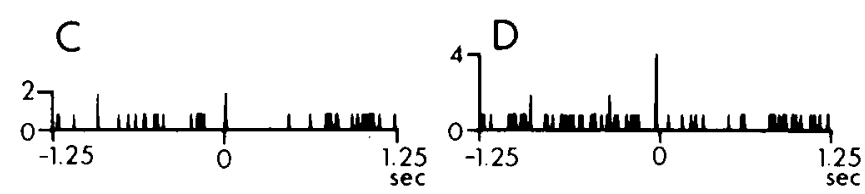

Figure 3. Different cross-correlation ( $X$ CORR) histograms illustrating complicated patterns of inhibitory interactions among adjacent 5-HT neurons in the DRN. $A$ shows synchrony near the origin. This would occur when both neurons receive a shared excitatory or inhibitory input from a common source. $B$ illustrates synchrony and "direct inhibition." This would occur when one cell inhibits the other and, at the same time, both receive a shared input from a third neuron; this histogram shows that the inhibition can be extremely powerful and prolonged. $C$ and $D$ are examples of synchrony and "mutual inhibition." This would occur when two adjacent neurons inhibit each other and they also have a shared input. In the latter cases, the synchronized firing is not as marked as in $A$ or $B$. 
addition to the aforementioned synchronization and "direct inhibition" (Fig. $3, A$ and $B$ ), there were concurrent synchronization and "mutual inhibition" which would occur, for example, when two neurons inhibit each other and, at the same time, both receive synchronizing input from a common source (any mechanism that simultaneously modulates the firing patterns of both neurons). In Figure 3, panels $C$ and $D$ are two such examples.

In contrast to complex inhibitory interactions among adjacent 5-HT neurons, cross-correlograms generated from adjacent cell pairs consisting of one 5-HT cell and a non-5-HT neuron or non-adjacent 5-HT neuronal pairs usually failed to show any functional interaction (Fig. 4; Table I); the proportion of neuronal pairs showing functional interaction in the latter cases were significantly lower than adjacent 5-HT neuronal pairs $(p<0.005$ for both cases; Fisher's test of significance for $2 \times 2$ table).

\section{Discussion}

The autocorrelation histograms from 5-HT neurons in the DRN showed a pronounced rhythmicity or periodicity. This result is expected from the fact that 5-HT neurons have a slow, regular firing pattern which is seen both in vivo and in brain slices in vitro (Mosko and Jacobs, 1976). On this basis, it has been suggested that 5HT neurons may have a pacemaker or homeostatic function (see Aghajanian and Wang, 1978 for review). Consistent with this view, autocorrelation histograms of 5HT neurons in the DRN bears a striking resemblance to the shape of autocorrelation histograms obtained from computer-simulated pacemaker neurons (Perkel et al., 1967a). All the autocorrelations of 5-HT neurons showed a pronounced trough after a spike discharge. Recently, intracellular recordings from 5-HT cells in the DRN have shown that this post-spike inhibition is associated with a prolonged hyperpolarizing afterpotential following each spike (G. K. Aghajanian and C. P. Vander Maelen, manuscript in preparation).
A

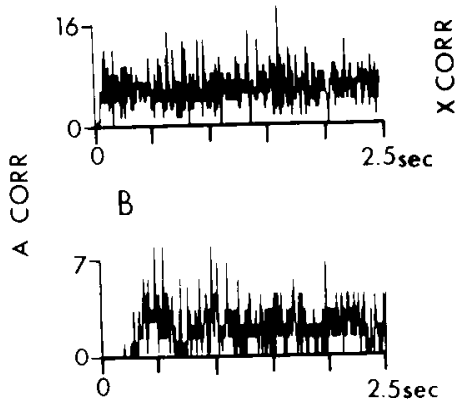

C

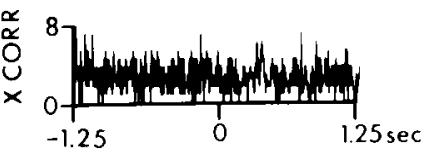

D

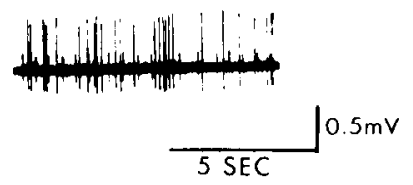

Figure 4. An example of autocorrelograms ( $A C O R R$ ), a cross-correlogram ( $X C O R R$ ), and a segment of spike trains of adjacent neuron pairs consisting of one 5-HT cell and one non5-HT cell. $A$ and $B$ are autocorrelation histograms obtained from a non-5-HT cell and a 5-HT neuron, respectively. $C$ is the cross-correlogram between the two cells. $D$ is a segment of their spike trains; the irregular firing pattern of the large non-5-HT cell contrasted with the slow, regular characteristic firing pattern of the small 5-HT neuron. As can be seen from $C$, there was no synchrony, direct inhibition, or mutual inhibition evident from the cross-correlogram.
Cross-correlation analysis for simultaneously recorded adjacent 5-HT neuronal pairs disclosed a high probability of correlated discharges within a short time (less than 10 msec), indicating that adjacent 5-HT cells discharge almost synchronously. In addition to this synchronous firing pattern, adjacent 5-HT neurons often displayed the interaction pattern of direct inhibition or mutual inhibition. The inhibitory effect often was powerful and prolonged, reminiscent of the effect produced by antidromic stimulation of the ascending 5 -HT bundle (Wang and Aghajanian, 1977c, 1978). There are several possible interpretations of the synchronous firing pattern among adjacent 5-HT neurons. As has been suggested previously (Perkel et al., 1967b; Moore et al., 1970), synchronous firing could be the result of direct activation from one cell to another or the result of sharing a direct activation or inhibition from a third neuron. In general, direct or shared activation gives rise to a sharper cross-correlation peak than does shared inhibition. According to this analysis, the former mechanism would fit best the pattern of synchronization seen in the DRN. On the other hand, disinhibition seems to account for the highly synchronized firing pattern of hippocampal pyramidal cells during deep sleep (Noda et al., 1969). Similarly, synchronous firing among adjacent 5 -HT cells could be the result of disinhibition. 5-HT-containing neurons could exert an inhibitory influence simultaneously upon a number of adjacent 5-HT neurons via dendrodendritic junctions. It would be necessary for such inhibitory effects to be strong enough to over-ride random facilitatory volleys impinging upon the 5-HT neurons (e.g., resulting from sensory arousal in awake animals; Trulson and Jacobs, 1979). Inhibitory volleys exerted by the 5-HT neurons upon adjacent 5-HT cells then might produce tightly coupled fluctuations in membrane potential. As the inhibitory effect wanes, the membrane potential of adjacent 5-HT neurons would rebound simultaneously. Therefore, the mechanism of shared inhibition and simultaneous disinhibition could account for the phenomenon of highly correlated discharges among adjacent 5HT neurons. Support for this view comes from our previous studies which show that: (1) antidromic stimulation of the ascending 5-HT fiber bundle induces a marked inhibitory effect upon all 5-HT neurons in the DRN; the inhibition is dependent upon the integrity of 5-HT fibers (Wang and Aghajanian, 1977b, 1978) and (2) iontophoretic application of 5-HT directly onto 5-HT-containing neurons invariably produces inhibition (Aghajanian et al., 1972; Haigler and Aghajanian, 1974; Wang and Aghajanian, 1977b).

It is of particular interest that the functional interactions among 5-HT neurons were restricted primarily to adjacent neurons; cross-correlations generated from nonadjacent 5-HT neuronal pairs usually failed to show any interactions. The latter finding indicates that: (1) between two regular firing 5 -HT cells, the probability that correlated interaction could emerge by chance is very low and (2) functional interactions among adjacent 5HT neurons are not due to the effect of anesthesia. The results further suggest that physiological coupling in the DRN takes place within small clusters of $5-\mathrm{H}^{\mathrm{T}} \mathrm{T}$ cells rather than between 5-HT cells in distant parts of the nucleus. This observation may be related to the finding 
that no specialized 5-HT axon-dendritic or axon-somatic synaptic junctions occur in the DRN as judged by electron microscopic autoradiography with $5-\left[{ }^{3} \mathrm{H}\right] \mathrm{HT}$ (Descarries et ai., 1979; Baraban and Aghajanian, 1981). This apparent lack of 5-HT axonal inputs in the DRN is consistent with the failure to note axon collaterals of cells in the DRN by Golgi techniques (Diaz-Cintra et al., 1981). On the other hand, specialized dendrodendritic synaptic junction (Felten and Harrigan, 1980) and gap junction (Mollgard and Moller, 1975) have been reported in the DRN. It remains to be determined whether these or perhaps other anatomical features of the DRN underlie the physiological interactions observed in the present study.

Are there any functional interactions among $5-\mathrm{HT}$ and non-5-HT neurons in the DRN? Our preliminary study of three 5-HT and non-5-HT neuronal pairs showed no obvious correlated interactions. However, further studies are needed to arrive at a definitive conclusion.

In conclusion, cross-correlograms of simultaneously recorded neuronal pairs in the DRN suggested that there are several distinct patterns of inhibitory interactions among adjacent 5-HT neurons. These results are consistent with previous findings that the firing of $5-\mathrm{HT}$ neurons in the DRN is governed by powerful auto- and mutual regulatory mechanisms. Studies using intracellular recordings will be required for obtaining more explicit and detailed information on the synaptic interactions among 5-HT neurons.

\section{References}

Aghajanian, G. K., and H. J. Haigler (1974) L-Tryptophan as a selective histochemical marker for serotonergic neurons in single-cell recording studies. Brain Res. 81: 364-372.

Aghajanian, G. K., and R. Y. Wang (1978) Physiology and pharmacology of central serotonergic neurons. In Psychopharmacology: A Generation of Progress, M. A. Lipton, A. DiMascio, and K. F. Killiam, eds., pp. 171-183, Raven Press, New York.

Aghajanian, G. K., H. J. Haigler, and F. E. Bloom (1972) Lysergic acid diethylamide and serotonin: Direct actions on serotonin-containing neurons. Life Sci. 11: 615-622.

Aghajanian, G. K., R. Y. Wang, and J. Baraban (1978) Serotonergic and non-serotonergic neurons of the dorsal raphe: Reciprocal changes in firing induced by peripheral nerve stimulation. Brain Res. 153: 169-175.

Baraban, J. M., and G. K. Aghajanian (1981) Noradrenergic innervation of serotonergic neurons in the dorsal raphe: Demonstration by electron-microscopic autoradiography. Brain Res. 204: 1-11.

Belin, M. F., M. Aguera, M. Tappaz, A. McRae-Dequeurce, P. Bobillier, and P. F. Pujol (1979) GABA-accumulating neurons in the nucleus raphe dorsalis and periaqueductal gray in the rat: A biochemical and radioautographic study. Brain Res. 170: 279-297.

Bennett, J. L., and G. K. Aghajanian (1974) D-LSD binding to brain homogenates: Possible relationships to serotonin receptors. Life Sci. 15: 1935-1944.

Bennett, J. P., and S. H. Snyder (1975) Stereospecific binding of $d$-lysergic acid diethylamide (LSD) to brain membranes: Relationship to serotonin receptors. Brain Res. 94: 523-544.

Bennett, J. P., and S. H. Snyder (1976) Serotonin and lysergic acid diethylamide binding in rat brain membranes: Relationship to postsynaptic serotonin receptors. Mol. Pharmacol. 12: 373-389.
Dahlström, A., and K. Fuxe (1965) Evidence for the existence of monoamine-containing neurons in the central nervous system. I. Demonstration of monoamines in the cell bodies of brainstem neurons. Acta Physiol. Scand. Suppl. 232 62: 1-55.

Descarries, L., A. Beaudet, K. C. Watkins, and S. Garcia (1979) 'The serotonin neurons in nucleus raphe dorsalis of adult rat. Anat. Rec. 193: 520.

Diaz-Cintra, S., L. Cintra, T. Kemper, O. Resnick, and P. J. Morgane (1981) Nucleus raphe dorsalis: A morphometric Golgi study in rats of three age groups. Brain Res. 207: 1-16.

Donzanti, B. A., and R. O. Warwick (1980) Evidence for high affinity serotonin uptake in rat periaqueductal gray region. Fed. Proc. 39: 607.

Felten, D. L., and P. Harrigan (1980) Dendrite bundles in nuclei raphe dorsalis and centralis superior of the rabbit: A possible substrate for local control of serotonergic neurons. Neurosci. Lett. 16: 275-280.

Felten, D. L., S. C. Rightor, and R. G. Peterson (1980) Ultrastructural characteristics and relationships of serotonergic perikarya in nuclei raphe dorsalis and obscurus of the rabbit brain. Soc. Neurosci. Abstr. 6: 351.

Fillion, G. M. B., J. -C. Rousselle, M. -P. Fillion, D. M. Beaudoin, M. R. Going, J. -M. Deniau, and J. J. Jacob (1978) High-affinity binding of $\left[{ }^{3} \mathrm{H}\right] 5$-hydroxytryptamine to brain synaptosomal membranes: Comparison with $\left[{ }^{3} \mathrm{H}\right]$ lysergic acid diethylamide binding. Mol. Pharmacol. 14: 38-49.

Gamrani, H., A. Calas, M. F. Belin, M. Aguera, and J. F. Pujol (1979) High resolution radioautographic identification of ${ }^{3} \mathrm{H}-$ GABA labelled neurons in the rat nucleus raphe dorsalis. Neurosci. Lett.. 15: 43-48.

Haigler, H. J., and G. K. Aghajanian (1974) Lysergic acid diethylamide and serotonin: A comparison of effects on serotonergic neurons and neurons receiving a serotonergic input. J. Pharmacol. Exp. Ther. 188: 688-699.

Kerwin, R. W., and C. J. Pycock (1979) The effect of some putative neurotransmitters on the release of 5-hydroxytryptamine and $\gamma$-aminobutyric acid from slices of the rat midbrain raphe area. Neuroscience 4: 1359-1365.

Lovell, R. A., and D. X. Freedman (1976) Stereospecific receptor sites for $d$-lysergic acid diethylamide in rat brain: Effects of neurotransmitters, amine antagonists and other psychotropic drugs. Mol. Pharmacol. 12: 620-630.

McGeer, E. G., U. Schere-Singler, and E. A. Singh (1979) Confirmatory data on habenular projections. Brain Res. 168 : 375-376.

Mollgard, K., and M. Moller (1975) Dendrodendritic gap junctions: A developmental approach. Adv. Neurol 12: 79-89.

Moore, G. P., D. H. Perkel, and J. P. Segundo (1966) Statistical analysis and functional interpretation of neuronal spike data. Annu. Rev. Physiol. 15: 493-522.

Moore, G. P., J. P. Segundo, D. H. Perkel, and H. Levitan (1970) Statistical sign of synaptic interaction in neurons. Biophys. J. 10: 876-900.

Mosko, S. S., and B. L. Jacobs (1976) Recording of dorsal raphe unit activity in vitro. Neurosci. Lett. $2: 195-200$.

Noda, H., S. Manohar, and W. R. Adey (1969) Correlated firing of hippocampal neuron pairs in sleep and wakefulness. Exp. Neurol. 24: 232-247.

Perkel, D. H., G. L. Gerstein, and G. P. Moore (1967a) Neuronal spike trains and stochastic point processes. I. The single spike train. Biophys. J. 7: 391-418.

Perkel, D. H., G. L. Gerstein, and G. P. Moore (1967b) Neuronal spike trains and stochastic point processes. II. Simultaneous spike trains. Biophys. J. $7:$ 419-440.

Peroutka, S. J., and S. H. Snyder (1979) Multiple serotonin receptors: Differential binding of ${ }^{3} \mathrm{H}$-5-hydroxytryptamine ${ }^{3} \mathrm{H}$-lysergic acid diethylamide and ${ }^{3} \mathrm{H}$-spiroperidol. Mol. Pharmacol. 16: 687-699. 
Shaskan, E., and S. H. Snyder (1970) Kinetics of ${ }^{3}$ H-5-hydroxytryptamine uptake into synaptosomes from different regions of rat brain. J. Pharmacol. Exp. Ther. 175: 404-418.

Steinbusch, H. W. M., D. van der Kooy, A. A. J. Verhofstad, and A. Pellegrino (1980) Serotonergic and non-serotonergic projections from the nucleus raphe dorsalis to the caudateputamen complex in the rat, studied by a combined immunofluorescence and fluorescent retrograde axonal labeling technique. Neurosci. Lett. 19: 137-142.

Thomas, R. C., and V. J. Wilson (1965) Precise localization of Renshaw cells with a new marking technique. Nature 206: 211-213.

Trulson, M. E., and B. L. Jacobs (1979) Raphe unit activity in freely moving cats: Correlation with level of behavioral arousal. Brain Res. 163: 135-150.

Wang, R. Y., and G. K. Aghajanian (1977a) Inhibition of neu- rons in the amygdala by dorsal raphe stimulation: Mediation through a direct serotonergic pathway. Brain Res. 120: 83102.

Wang, R. Y., and G. K. Aghajanian (1977b) Recording of single unit activity during electrical stimulation and microiontophoresis: A method of minimizing stimulus artifacts. Electroencephalogr. Clin. Neurophysiol. 43: 434-437.

Wang, R. Y., and G. K. Aghajanian (1977c) Antidromically identified serotonergic neurons in the rat midbrain raphe: Evidence for collateral inhibition. Brain Res. 132: 186-193.

Wang, R. Y., and G. K. Aghajanian (1978) Collateral inhibition of serotonergic neurons in the rat dorsal raphe nucleus: Pharmacological evidence. Neuropharmacology 17: 819-825. Young, W. S., III, and M. J. Kuhar (1980) Serotonin receptor localization in rat brain by light microscopic autoradiography. Eur. J. Pharmacol. 62: 237-239. 